ANL-HEP-PR-94-58

November 1994

\title{
Aspects of four-jet production in polarized proton-proton collisions
}

\author{
S. P. Fraser and S. T. Fraser" \\ Department of Physics and Astronomy \\ Sonoma State University \\ Rohnert Park, CA 94928 USA \\ and \\ R. W. Robinett \\ High Energy Physics Division \\ Argonne National Laboratory \\ Argonne, IL 60439 USA \\ and \\ Department of Physics \\ Penn State University \\ University Park, PA 16802 USA \\ e-mail:rq9@psuvm.psu.edu
}

\begin{abstract}
We examine the intrinsic spin-dependence of the dominant $g g \rightarrow g g g g$ subprocess contribution to four-jet production in polarized proton-proton collisions using helicity amplitude techniques. We find that the partonic level, longitudinal spin-spin asymmetry, $\hat{a}_{L L}$, is intrinsically large in the kinematic regions probed in experiments detecting four isolated jets. Such events may provide another qualitative or semi-quantitative test of the spin-structure of QCD in planned polarized $p p$ collisions at RHIC.
\end{abstract}

\footnotetext{
${ }^{1}$ Address as of Sept. 1, 1994, Univ. Heidelberg, D-W-6900, Heidelberg 1 GERMANY
} 
The prospects for a comprehensive program of polarized proton-proton collisions at collider energies at RHIC [1, 2], culminating in the approved experiment R5 [3], has motivated a large number of studies of the spin-dependence of many standard model processes and their sensitivity to polarized parton distributions. Many processes which already have been well-studied, both theoretically and experimentally, at existing unpolarized hadron colliders, have been reexamined in the context of a physics program dedicated to the extraction of the spin-dependent quark, antiquark, and gluon distributions and tests of the spin-dependence of the basic hard scattering processes of QCD and the electroweak sector.

Familiar processes such as direct photon production [4, 5, 6] and Drell-Yan lepton pair production [6, 7] (including $W$ and $Z$ production [6, 8]) are known to be sensitive to the longitudinally polarized gluon and sea-quark content of the proton respectively. (We will henceforth take polarized to mean longitudinal polarization; transverse polarization effects will also be studied at RHIC but will only be briefly discussed here.) Calculations of the radiative corrections to the spin-dependent cross-sections for these processes have even appeared [9, 10] and confirm the general conclusions of leadingorder results. Jet production has also been extensively studied at lowest order [5, 11] and the technology for the efficient calculation of the NLO corrections to helicity amplitudes for jet production is now well-known [12] although a detailed analysis of the spin-dependent radiative corrections to jet cross-sections has not yet been performed. Taken together, these processes already provide the basis for a substantial experimental program using the two planned RHIC detectors [3], STAR and PHENIX, which are complementary in their physics capabilities.

A wide variety of other processes has also been studied at leading-order in the context of the RHIC spin program including heavy quark production [13, 14, quarkonium 
production in different kinematic regions [15, 16, 17], three-jet production [18], and double-photon $(\gamma \gamma)$ production [19]. All of these processes have been studied experimentally, to varying degrees, in collider energy unpolarized $p p$ or $p \bar{p}$ collisions and seem to agree reasonably well with theoretical expectations. Some other processes whose spin-dependence has also been discussed, such as two-jet plus direct photon [18] and $\psi+\gamma$ [20], have yet to be measured but may be detectable for the first time given the large luminosity and energies (up to $2 \times 10^{32} \mathrm{~cm}^{-2} \mathrm{~s}^{-1}$ and $\sqrt{\mathrm{s}}=500 \mathrm{GeV}$ ) possible at RHIC and the nature of the proposed detectors.

Just as with single inclusive jet, di-jet, and three-jet production, four-jet production has been observed at hadron colliders ranging from the ISR [21, 22] $(\sqrt{s}=63 \mathrm{GeV})$ to the CERN $S p \bar{p} S$ 23] $(\sqrt{s}=630 \mathrm{GeV})$ and most recently at the TEVATRON [24] $(\sqrt{s}=1.8 \mathrm{TeV})$. In the last two cases, comparisons of data to leading order QCD predictions for various shape and angular variables have been made and reasonable agreement is found. Besides providing another test of standard QCD, this process has the additional feature that it has also been widely discussed as a possible arena in which to study double-parton scattering [25, 26], an effect which is expected to appear at sufficiently high energies.

Because the $2 \rightarrow 4$ subprocesses leading to four-jet production are proportional to $\alpha_{S}^{4}$, changes in the value of $\alpha_{S}$ used and uncertainties in the choice of $Q^{2}$ scale (i.e. lack of knowledge of the next order QCD corrections) make the prediction of the absolute rates difficult. This coupled with the lower rates compared to two- and threejet production and the increasing difficulty of unambiguously defining $n$ isolated jets makes this process, at present, a semi-quantitative or qualitative test of QCD. Because rapid progress is being made in the calculation of the NLO matrix elements for two- 
and three-jet production [12] with hope held out for the eventual evaluation of the necessary radiative corrections for the $2 \rightarrow 4$ subprocesses as well [27, this situation may well change in the not-too-distant future.

Motivated by the large rates possible for many hadron processes at the high luminosity polarized $p p$ RHIC facility, in this note we briefly discuss the spin-dependence of the dominant QCD subprocess contributing to such four-jet production, namely $g g \rightarrow g g g g$; the analysis presented here thus extends and complements the discussion of the spin-dependence of three-jet production given in Ref. [18]. Because existing limits [23, 24] imply that double-parton scattering will not be important at any RHIC energy, we will discuss only the spin-dependence of $2 \rightarrow 4$ processes.

We follow the analysis of four-jet production at TEVATRON energies as given in Ref. [26] with some minor differences. We consider only the dominant $g g \rightarrow g g g g$ subprocess and because we are interested in the spin-dependence we use the exact expressions for the matrix elements for this process instead of relying on any approximation technique; specifically, we use the compact expressions for the necessary helicity amplitudes in Ref. 28] and Ref. [29]. In the calculation of the appropriate cross-sections, we modify the cuts of Ref. 26] Set 2 (motivated by the smaller RHIC energy of $\sqrt{s}=500 \mathrm{GeV}$ ) and insist that $p_{T}>15 \mathrm{GeV}$ and $|\eta| \leq 0.8$ for each jet and that $\cos \left(\theta_{i j}\right) \leq 0.643$ for each jet pair. Finally, we insist that there is a minimum total transverse energy of $E_{T} \geq 70 \mathrm{GeV}$. For this leading-order calculation, we use the updated parton distributions of Duke-Owens (in this case, only the gluon distribution of Set I is required) of Ref. [30].

The resulting differential cross-section as a function of transverse momentum, $d \sigma / d p_{T}$ versus $p_{T}$, (with four entries per event) is shown as the dot-dash curve in Fig. 1 and 
has a very similar shape as the corresponding plot (Fig. 2 (a)) in Ref. [26]. The spin-dependent cross-sections

$$
\frac{d \Delta \sigma}{d p_{T}} \equiv \frac{1}{2}\left(\frac{d \sigma(++)}{d p_{T}}-\frac{d \sigma(+-)}{d p_{T}}\right)
$$

where $(++),(+-)$ refers to the helicities of the incident protons are also shown in Fig. 1 for two choices of the polarized gluon distribution. The spin-dependent gluon distribution, conventionally written as $\Delta G\left(x, Q^{2}\right) \equiv G_{+}\left(x, Q^{2}\right)-G_{-}\left(x, Q^{2}\right)$ (where $+/-$ refers to parton helicity in the same/opposite direction as the parent proton helicity), is assumed, for simplicity, to have the form $\Delta G\left(x, Q^{2}\right)=x^{\alpha} G\left(x, Q^{2}\right)$. The two choices $\alpha=1$ (solid curve) and $\alpha=0.25$ (dashed curve) correspond to an integrated gluon contribution $\Delta G=\int \Delta G\left(x, Q^{2}\right) d x$ equal to 0.5 and 4.5 respectively and thus bracket the expectations for a 'normal' to 'large' gluonic contribution to the total proton spin.

Using the $g g \rightarrow g g g g$ matrix elements and these cuts, we have calculated differential distributions in the other kinematic variables discussed in Ref. [26], namely $p_{\text {out }}, \phi_{\text {min }}$, and $\cos \left(\theta_{23}^{*}\right)$. The transverse momentum out of the plane passing through the beam and the jet of largest $p_{T}$, i.e. $p_{\text {out }}$, is defined via

$$
p_{\text {out }} \equiv \frac{1}{2} \sum_{i}\left|p_{\text {out }}^{i}\right|
$$

while $\phi_{\min }$ is the minimum angle in the transverse plane between the largest $p_{T}$ jet and the other three jets and finally $\cos \left(\theta_{23}^{*}\right)$ is the cosine of the angle between the second and third most energetic jets in the four-jet center-of-mass. Differential distributions in each of these quantities look very similar to the corresponding plots in Fig. 2(a)-(d) in Ref. 26. 
The measurable spin-spin asymmetry in any observable quantity, defined by

$$
A_{L L}=\frac{d \sigma(++)-d \sigma(+-)}{d \sigma(++)+d \sigma(+-)}
$$

is determined by both the partonic level spin-dependence of the underlying hard scattering,

$$
\hat{a}_{L L}=\frac{d \hat{\sigma}(++)-d \hat{\sigma}(+-)}{d \hat{\sigma}(++)+d \hat{\sigma}(+-)}
$$

and the magnitude of the polarized parton distributions since

$$
A_{L L} d \sigma=\sum_{i, j} \int d x_{a} \int d x_{b} d \hat{\sigma} \hat{a}_{L L} \Delta f_{i}\left(x_{a}, Q^{2}\right) \Delta f_{j}\left(x_{b}, Q^{2}\right)
$$

Using the polarized parton distributions mentioned above and the exact $2 g \rightarrow 4 g$ matrix elements, we find the observable asymmetries for the four variables $p_{T}, p_{\text {out }}$, $\phi_{\text {min }}$, and $\cos \left(\theta_{23}^{*}\right)$ shown in Fig. 2(a)-(d). The increasing value of $A_{L L}$ with larger values of $p_{T}$ (Fig. 2(a)) and $p_{\text {out }}$ (Fig. 2(b)) is reminiscent of similar effects in two-jet [5, 11] and three-jet [18] production and simply reflects the increase in gluon polarization for large $x$ (where, of course, the cross-sections are very small). Figs. 2(a) and (b) also show that the intrinsic spin-spin asymmetry of the hard-scattering subprocess, $\hat{a}_{L L}$, is large; we estimate that the average value of the partonic level spin-spin asymmetry in the configurations measured in four-jet production is roughly $\left\langle\hat{a}_{L L}\right\rangle \sim 0.8$ which is even larger then the corresponding value of $\left\langle\hat{a}_{L L}\right\rangle \sim 0.7$ found for the $g g \rightarrow g g g$ contribution to three-jet production. We have also checked explicitly that when one gluon is allowed to be soft the resulting partonic level spin-spin asymmetries reduce to those for three-jet production derived in Ref. [18]. On the other hand, the angular variables we have studied show little variation for a given set of polarized distribution functions as seen in Fig. 2 (c) and (d); this is also consistent with earlier results on 
spin-dependence in three-jet events. These results reaffirm the fact observed in the case of two- and three-jet production, namely that once one is in the kinematic regime required for the isolation of jets, the intrinsic longitudinal spin-spin asymmetries for gluon initiated processes is almost maximally large and varies little with the relative orientation of the jets.

One can also consider transverse spin effects in jet physics but the situation is far less diverse. The quarks and antiquarks (and not the gluons) carry the 'transversity' [31] so that only $q q$ or $q \bar{q}$ initiated processes in transversely polarized $p p$ collisions need be considered. It has been known for some time [32] that the partonic level transverse spin-spin asymmetries, $\hat{a}_{T T}$, for the relevant $2 \rightarrow 2$ processes vanish in the case of unlike quark scattering via $q q^{\prime} \rightarrow q q^{\prime}$ and are much smaller (roughly a factor of 10) than the corresponding longitudinal asymmetry $\left(\hat{a}_{L L}\right)$ for like-quark scattering via $q q \rightarrow q q$ due to a color factor. An identical pattern is seen in the transverse spin-spin asymmetries for three-jet production via $q q^{\prime} \rightarrow q q^{\prime} g$ and $q q \rightarrow q q g$ [33]. We expect a similar situation in the four-jet case for the processes $q q^{\prime} \rightarrow q q^{\prime} g g, q q \rightarrow q q g g$ and $q q^{\prime} \rightarrow q q^{\prime} q q$. Since these processes only form a significant part of the four-jet crosssection at the very largest values of $p_{T}$ where the cross-sections are unmeasureably small, we expect no significant transverse spin dependence in such processes.

\section{Acknowledgments}

One of us (RWR) gratefully acknowledges the hospitality of Argonne National Laboratory where this work was completed. This work was supported, in part, by a grant from the National Science Foundation under the Research Experiences for Undergraduates (REU) program (SPF, STF) at Penn State, by Argonne National Laboratory under the Faculty Research Participation Program (RWR), and by the U. S. Department of Energy, Division of High Energy Physics, Contract W-31-109-ENG-38 (RWR). 


\section{References}

[1] G. Bunce et al., Particle World 3, 1 (1992).

[2] Proceedings of the Polarized Collider Workshop, Penn State University 1990, edited by J. Collins, S. Heppelmann, and R. W. Robinett, AIP Conf. Proceedings No. 223 (AIP, New York, 1991).

[3] Joint RSC-STAR-PHENIX Collaboration, "Proposal on spin physics using the RHIC polarized collider", (August 14, 1992); update (Sept. 2, 1993); approved October 1993 as Brookhaven experiment R5.

[4] E. L. Berger and J. Qiu, Phys. Rev. D40, 778 (1989); J. Qiu in [2], p. 162.

[5] See, e.g., C. Bourrelly, J. Ph. Guillet, and J. Soffer, Nucl. Phys. B361, 72 (1991).

[6] P. Chiapetta, P. Colangelo, J. -Ph. Guillet, and G. Nardulli, Z. Phys. C59 (1993) 629.

[7] H. -Y. Cheng and S. -N. Lai, Phys. Rev. D41, 91 (1990); S. Gupta, D. Indumathi, and M. V. N. Murthy, Z. Phys. C42, 493 (1989): (E) C44, 356 (1989); E. Leader and K. Sridhar, Phys. Lett. B311, 324 (1993).

[8] C. Bourrely and J. Soffer, Phys. Lett. B314, 132 (1993); Nucl. Phys. B243, 329 (1994).

[9] For radiative corrections to spin-dependent direct photon production, see A. P. Contogouris, B. Kamal, Z. Merebashvili, and F. V. Tkachov, Phys. Lett. B304, 329 (1993); Phys. Rev. D48, 4092 (1993); L. E. Gordon and W. Vogelsang, Phys. Rev. D48, 3136 (1993); D50, 1901 (1994).

[10] For radiative corrections to spin-dependent Drell-Yan production, see P. Ratcliffe, Nucl. Phys. B223, 45 (1983); P. Mathews and V. Ravindran, Mod. Phys. Lett. A7, 2695 (1992); A. Weber, Nucl. Phys. B382, 63 (1992).

[11] H. -Y. Cheng, S. -R. Hwang, and S. -N. Lai, Phys. Rev. D42, 2243 (1990); P. Chiapetta and G. Nardulli, Z. Phys. C51, 435 (1991); Z. Kunszt, Phys. Lett. B218 (1989) 243.

[12] Z. Bern and D. A. Kosower, Phys. Rev. Lett. 66, 1669 (1991); Nucl. Phys. B379, 451 (1992); Z. Bern, L. Dixon, and D. A. Kosower, Phys. Rev. Lett. 70, 2677 (1993).

[13] A. P. Contogouris, S. Papadopoulos, and B. Kamal, Phys. Lett. 246B, 523 (1990)

[14] M. Karliner and R. W. Robinett, Phys. Lett. B324, 209 (1994).

[15] J. Cortes and B. Pire, Phys. Rev. D38, 3586 (1988).

[16] M. A. Doncheski and R. W. Robinett, Phys. Lett. B248,188 (1990).

[17] R. W. Robinett, Phys. Rev. D43, 113 (1991). 
[18] M. A. Doncheski, R. W. Robinett, and L. Weinkauf, Phys. Rev. D44, 2717 (1991).

[19] M. A. Doncheski and R. W. Robinett, Phys. Rev. D46, 2011 (1992).

[20] M. A. Doncheski and C. S. Kim, Phys. Rev. D49, 4463 (1994).

[21] (ACDHW collaboration) A. Breakstone et al., Z. Phys. C23, 1 (1984).

[22] (AFS Collaboration) T. Åkesson et al., Z. Phys. C34 (1987) 163.

[23] (UA2 Collaboration) V. Cavasinni, "Proceedings of of the 4th Meeting of the DPF of the APS", University of Connecticut, 1988, edited by K. Haller (World Scientific, Singapore, 1988), p. 452; M. Wunsch, "Proceedings of the XXIIIrd Rencontres de Moriond", Savoie, France, 1988, edited by J. Tran Thanh Van (Edition Frontieres, 1988), p. 329.

[24] (CDF Collaboration) L. J. Keeble, "Proceedings of the DPF '92 Meeting", Fermi National Accelerator, Batavia, IL, 1992, edited by C. Albright, P, Kasper, R. Raja, and J. Yoh (World Scientific, Singapore, 1992), p. 1002; "Proceedings of the XXVIIth Rencontres de Moriond", Savoie, France, 1992, edited by J. Tran Thanh Van (Edition Frontieres, 1992), p. 135.

[25] B. Humpert and R. Odorico, Phys. Lett. 154B, 211 (1985); Ll. Ametller, N. Paver,and D. Treleani, Phys. Lett. 169B, 289 (1986); C. J. Maxwell, Phys. Lett. 192B, 190 (1987).

[26] M. Mangano, Z. Phys. C42, 331 (1989).

[27] Z. Bern, L. Dixon, D. C. Dunbar, and D. A. Kosower, to appear in "Proceedings of QCD94", Montpelier, France, July 1994.

[28] We have used the $\mathcal{C}$-function expressions in J. G. Kuijf, $\mathrm{PhD}$ thesis, University of Leiden, 1991, except for $\mathcal{C}^{++-+--}$and $\mathcal{C}^{+-+-+-}$, which do not appear to satisfy the necessary reflective and cyclic properties.

[29] We have used two expressions in F. A. Berends and W. Giele, Nucl. Phys. B294, 700 (1987); see also the equivalent formulation given by M. Mangano, S. Parke, and Z. Xu, Nucl. Phys. B298, 653 (1988).

[30] J. F. Owens, Phys. Lett. B266, 126 (1991).

[31] R. L. Jaffe and X. -D. Ji, Phys. Rev. Lett. 67, 552 (1991); X. -D. Ji, Phys. Lett. B284, 137 (1991)

[32] K. Hidaka, E. Monsay, and D. Sivers, Phys. Rev. D19, 1503 (1979).

[33] R. W. Robinett, Phys. Rev. D45, 2563 (1992). 


\section{Figure Captions}

Fig. 1. The solid and dashed curves show the spin-dependent, differential cross-section, $d \Delta \sigma / d p_{T}(n b / G e V)$ versus $p_{T}(G e V)$, for four-jet production from the $g g \rightarrow g g g g$ subprocess with the cuts discussed in the text; the solid (dashed) curves correspond to a polarized gluon distribution given by $\Delta G\left(x, Q^{2}\right)=x^{\alpha} G\left(x, Q^{2}\right)$ with $\alpha=1(0.25)$. The dot-dash curve is the unpolarized differential cross-section $d \sigma / d p_{T}(n b / G e V)$ versus $p_{T}(G e V)$ with the same assumptions.

Fig. 2. The spin-spin asymmetry, $A_{L L}$, in various differential cross-sections for four-jet production. The plots correspond to asymmetries in differential distributions for (a) $p_{T}$, (b) $p_{\text {out }},(\mathrm{c}) \phi_{\text {min }}$, and (d) $\cos \left(\theta_{23}^{*}\right)$. The solid (dashed) curves in each plot correspond to polarized gluon distributions given by $\Delta G\left(x, Q^{2}\right)=x^{\alpha} G\left(x, Q^{2}\right)$ with $\alpha=1(0.25)$. 
This figure "fig1-1.png" is available in "png" format from: http://arxiv.org/ps/hep-ph/9411359v1 


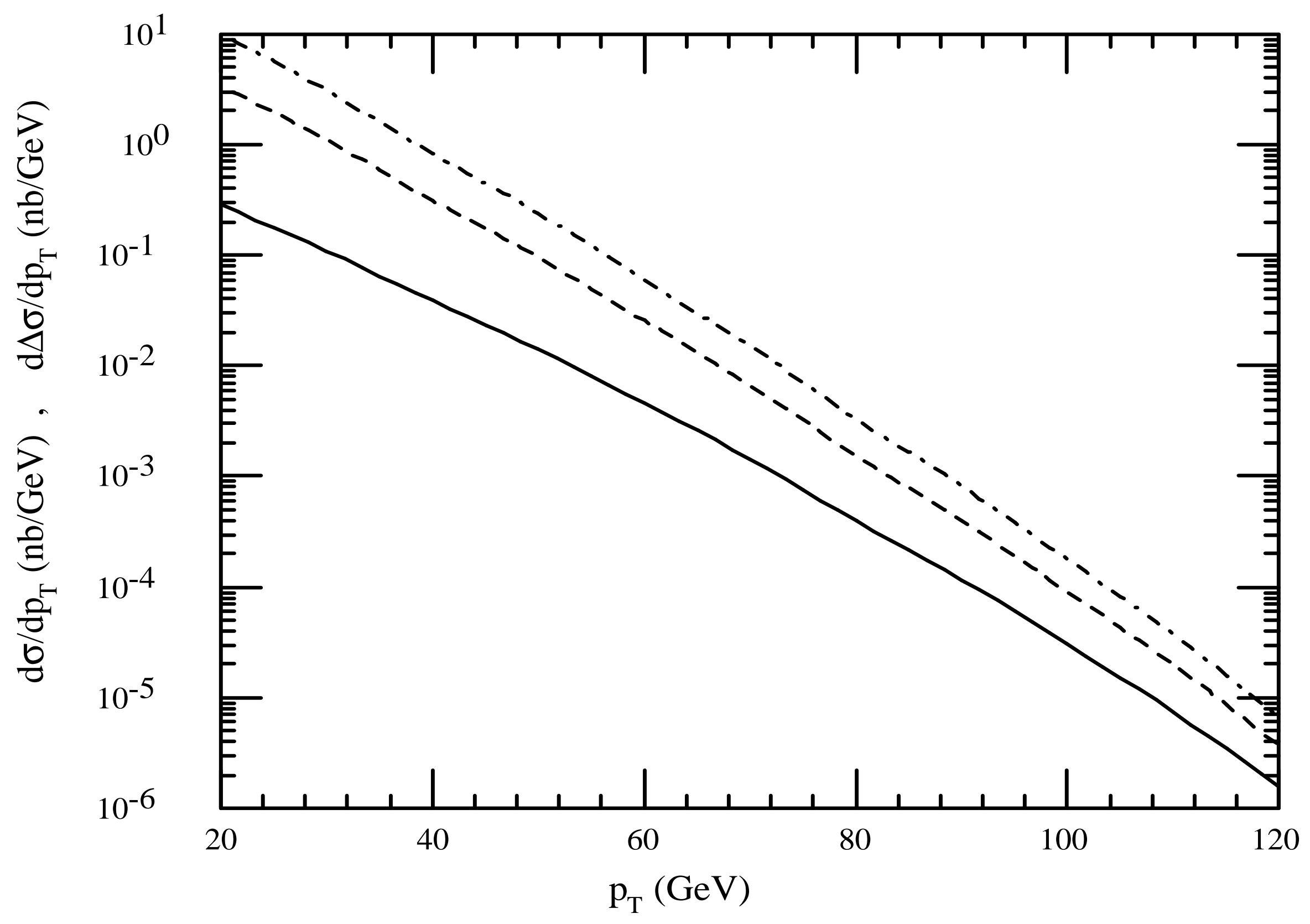


This figure "fig1-2.png" is available in "png" format from: http://arxiv.org/ps/hep-ph/9411359v1 

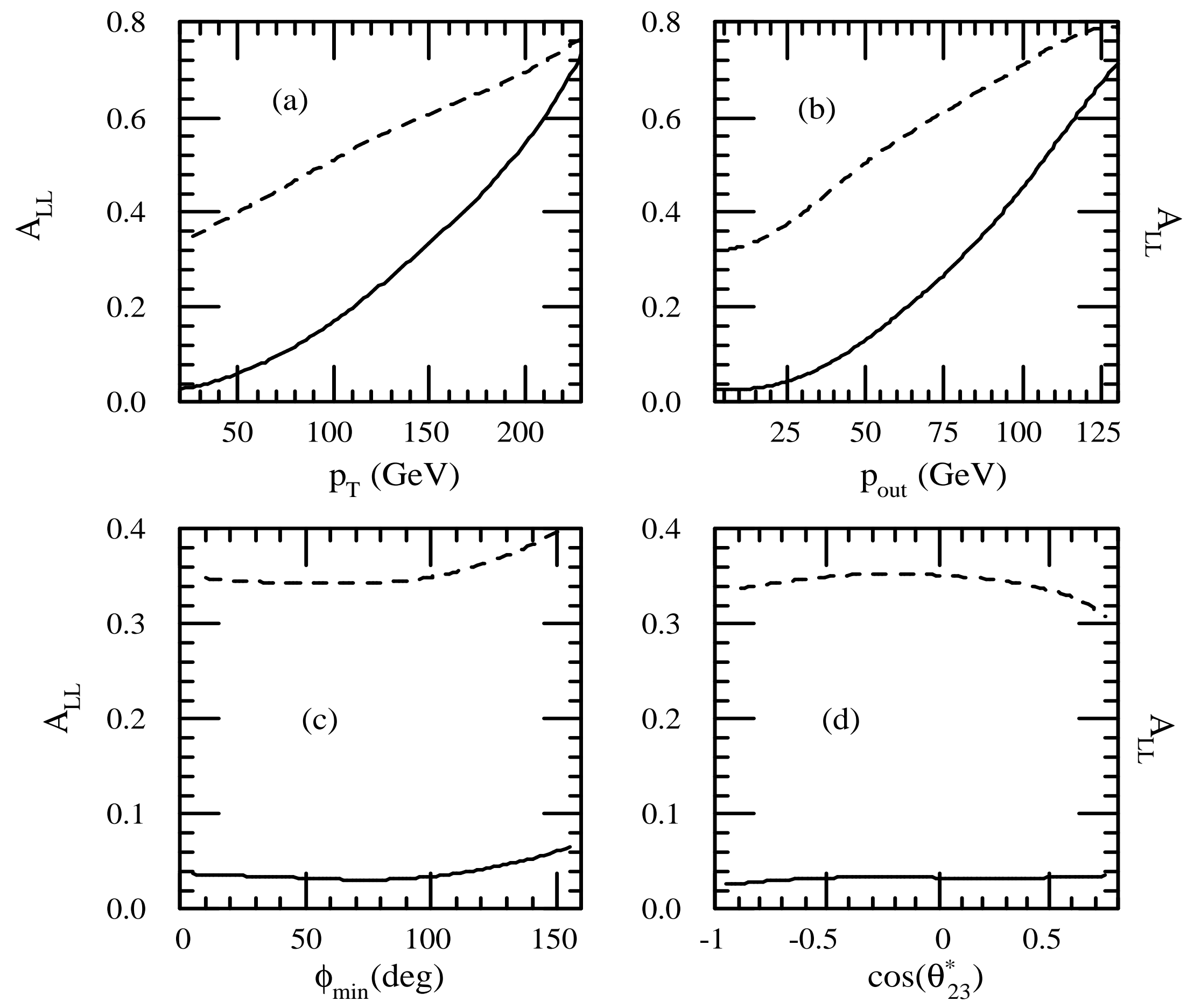